\title{
EFECTIVIDAD DE LA MARMOLINA COMO MINERAL INERTE EN EL CONTROL DE Hypothenemus hampei (FERRARI) (COLEOPTERA: CURCULIONIDAE) DURANTE LA CONSERVACIÓN DE SEMILLAS
}

\section{EFFECTIVENESS OF MARMOLINA AS AN INERT MINERAL IN THE CONTROL OF Hypothenemus hampei (FERRARI) (COLEOPTERA: CURCULIONIDAE) DURING STORAGE OF COFFEE SEEDS}

\author{
Yandy Rodríguez Ledesma ${ }^{1}$, Claribel Suárez Pérez², Justo Antonio Rojas Rojas³ , Raúl Mirabal García ${ }^{4}$, \\ Yasel López López ${ }^{5}$, Erismany Molina Moreno ${ }^{6}$.
}

\begin{abstract}
${ }^{1}$ Ingeniero Agrónomo. Máster en Ciencias Agrícolas. Centro Universitario Fomento. Máximo Gómez 103. Fomento, Sancti Spíritus. Cuba. CP: 62500. E-mail: yledesma@uniss.edu.cu; ${ }^{2}$ Ingeniero Agrónomo, Doctora en Ciencias Pedagógicas. .Universidad de Sancti Spíritus "José Martí Pérez" .Ave de los Mártires. Número 360, Sancti Spíritus. CP 60100. E-mail: claribel@uniss.edu.cu; ${ }^{3}$ Ingeniero Agrónomo, Doctor en Ciencias Agrícolas Universidad de Sancti Spíritus "José Martí Pérez" .Ave de los Mártires. Número 360, Sancti Spíritus. CP 60100 e-mail: justo@uniss.edu.cu; ${ }^{4}$ Ingeniero Agrónomo. Máster en Ciencias Agrícolas. Estación de Protección de plantas (EPP).Carretera circunvalante, Fomento, Sancti Spíritus. CP: 62500; ${ }^{5}$ Ingeniero Agrónomo, Máster en Ciencias Agrícolas Facultad de Ciencias Agropecuarias. Universidad de Sancti Spíritus “José Martí Pérez" .Ave de los Mártires. Número 360, Sancti Spíritus. CP 60100. E-mail: yasel@uniss.edu.cu; ${ }^{6}$ Ingeniero Agrónomo, Máster en Ciencias Agrícolas. Facultad de Ciencias Agropecuarias. Universidad de Sancti Spíritus "José Martí Pérez" .Ave de los Mártires. Número 360, Sancti Spíritus. CP 60100.
\end{abstract}

Rev. U.D.C A Act. \& Div. Cient. 18(2): 351-358, Julio-Diciembre, 2015

\section{RESUMEN}

Con el objetivo de evaluar la efectividad de la marmolina, como polvo inerte en el control de la broca del café, Hypothenemus hampei (Ferrari) (Coleoptera: Curculionidae), durante el período de conservación de las semillas, se determinó el efecto anti-insecto, mortalidad, porcentaje de pérdida de peso y germinación. Se utilizó un diseño completamente al azar, en un arreglo de 4 tratamientos y 3 dosis. Los tratamientos, se evaluaron en dosis de 0,46g; $0,81 \mathrm{~g} ; 1,74 \mathrm{~g}$ y un control (sin polvo), con tres réplicas, cada uno. Se realizó un análisis de varianza en el paquete estadístico statgraphics versión 5.0. Para el cálculo de mortalidad, todos los datos obtenidos fueron transformados en arc sen $(x / 100)^{0,5}$. La marmolina, se adhiere con facilidad a la cutícula del insecto, debido a su alta superficie específica $14 \mathrm{~m}^{2} \mathrm{~g}^{-1}$ y actúa matándolo, mediante un proceso físico de desecación y debido a su composición química. Según los resultados, se obtuvieron valores de mortalidad de $100 \%$, a los 7 días, con las tres dosis empleadas. Entre mayor es la dosis de marmolina utilizada en las semillas menor será el ataque del insecto a las mismas y, entonces, menor el porcentaje de peso de la muestra. La marmolina aplicada a las semillas, para el control de la broca del café, no afecta la germinación de las semillas almacenadas, por un periodo de 10 meses.

Palabras clave: Café, germinación, polvo mineral, marmolina, semillas almacenadas.

\section{SUMMARY}

In order to assess the effectiveness of marmolina as inert dust in the control of the coffee berry borer, Hypothenemus hampei (Ferrari) (Coleoptera: Curculionidae) during the period of seed storage, its anti-insect effect, borer mortality, seed percentage of weight loss and germination was determined. The design was completely randomized 4 treatments and 3 doses. Treatments were evaluated in dose of $0.46 \mathrm{~g} ; 0.81 \mathrm{~g} ; 1.74 \mathrm{~g}$ and a control (without dust) with three replicates each one. An analysis of variance was performed with the statistical package Statgraphics version 5.0. To calculate mortality, all data were transformed into arcsin (x $/ 100)^{0.5}$. Marmolina sticks to the insect cuticle due to its high specific surface $14 \mathrm{~m}^{2} \cdot \mathrm{g}-{ }^{1}$ and works by killing the insect by physical drying process due to the presence of various 
chemical. According to the results a $100 \%$ mortality at 7 days after the treatment was obtained with the three doses. Marmolina applied to seeds for the control of the coffee berry borer does not affect the germination of seeds stored for a period of 10 months.

Key words: Coffee, germination, mineral powder, marmolina, stored seeds.

\section{INTRODUCCIÓN}

La broca del café Hypothenemus hampei (Ferrari) (Coleoptera: Curculionidae) constituye el principal problema fitosanitario del cafeto en todo el mundo (Bustillo et al. 2008; Díaz-Vicente et al. 2014) y, actualmente, para Cuba. Según, Bustillo (2005), Bustillo-Pardey (2006), Vázquez et al. (2005) y Cintrón-Valdés \& Grillo-Ravelo (2009), su daño fundamental radica en que para establecerse y reproducirse, desarrolla galerías en el interior de los frutos, afectando, tanto el rendimiento como la calidad del producto agrícola y, de manera específica, compromete la germinación, así como la viabilidad de la semilla, cuando ésta es afectada, durante el periodo de conservación. Al respecto, Pérez et al. (2013) mencionan que el Manejo Integrado de la Broca (MIB) es una estrategia o plan de acción para reducir el daño y las pérdidas económicas que ocasiona la plaga al cultivo; por tanto, el éxito en el manejo y en el control de la broca del café depende de la acción conjunta, entre una buena asesoría técnica, el desarrollo de las medidas de control preventivas por parte del productor y, según Díaz-Vicente et al. (2014), de la investigación de alternativas viables en la producción.

Arrubla et al. (2009) plantean que, actualmente, para lograr un mayor efecto de los insecticidas y reducir su impacto ambiental negativo, existe la tendencia a disminuir su uso en la aplicación, para el control de las plagas; sin embargo, en este sentido, la alternativa más viable es la de utilizar productos naturales, como polvos vegetales, aceites naturales o polvos minerales inertes, específicamente, sobre las semillas para el control de plagas, durante el almacenamiento del fruto.

Muchos son los autores que se refieren a la importancia de garantizar la viabilidad de la semilla en este periodo (Levy et al. 1989; Arizaleta et al. 2002) y, otros, a aspectos, como la humedad y las condiciones de envases (Ortuño \& Echandi, 1980; Barboza \& Herrera, 1990; Días \& Barrios, 1993), descuidando el hecho que el ataque de plagas insectiles es un elemento muy importante a tener en cuenta, debido a que afectan y complican los diferentes fines que se pudiesen dedicar para la misma, como son su venta o posterior consumo.

Existe poca información referente a la conservación y al almacenamiento de las semillas de cafeto y, sobre todo, con polvos minerales; sin embargo, en la literatura se reseña, como característica importante (Arizaleta et al. 2005), que dichas semillas se deterioran rápidamente al ser almacenadas. Dada la importancia económica y social del cultivo del cafeto para Latinoamérica y otros países, conjuntamente con el alto deterioro que presentan las semillas durante el almacenamiento por la afectación de la broca del café y según Quispe-Condori et al. (2014), constituye razón suficiente para justificar un interés marcado hacia este cultivo y todo lo relacionado con el mismo.

Según, Urribarr et al. (2014), el café es uno de los productos agrícolas cultivado en gran extensión en el mundo. En Cuba, se considera de gran importancia, no solo por su influencia en el producto interno bruto sino por su relevancia social, al constituir fuente de sustento de un amplio segmento de la población (Pérez et al. 2013).

En la actualidad, existe una tendencia de carácter mundial hacia la búsqueda de métodos alternativos, que proporcionen resultados en el control de insectos en almacenes y que disminuyan las pérdidas económicas, pero sin los riesgos que implica el uso de plaguicidas de alta toxicidad, para el hombre y el medio ambiente (Silva et al. 2004), como la aplicación de diferentes polvos inertes.

Por lo anteriormente expuesto, constituyó objetivo de la investigación, evaluar la efectividad de la marmolina en el control de Hypothenemus hampei (Ferrari) (Coleoptera: Curculionidae), como polvo mineral inerte, durante la conservación de semillas de café.

\section{MATERIALES Y MÉTODOS}

El trabajo, se realizó en el laboratorio de la Estación de Protección de Plantas (EPP), ubicada en el municipio Fomento, provincia Sancti Spíritus, Cuba Central.

Se utilizó un diseño completamente al azar, en un arreglo de 4 tratamientos y 3 dosis. Los tratamientos, se evaluaron en dosis de $0,46 \mathrm{~g} ; 0,81 \mathrm{~g} ; 1,74 \mathrm{~g}$ y un control (sin polvo), con tres réplicas, cada uno. Para el experimento, se utilizaron adultos de broca del café, recolectados en cosecha y clasificados en la EPP.

La marmolina, se obtuvo en el corta-bloques, ubicado en el municipio Fomento, Sancti Spíritus y perteneciente a la empresa Mármoles Centro (Cuba). La caracterización química del compuesto, se realizó en los laboratorios de la Empresa Geominera del Centro (Villa Clara, Cuba). El volumen de producción de marmolina de la empresa en este municipio es de $1,56 \mathrm{~m}^{3}$ semanales, aproximadamente.

Debido a que no se encontraron antecedentes de investigaciones previas acerca del uso de la marmolina en la conser- 
vación de semillas, se decidió adoptar, para nuestros estudios, la metodología propuesta por autores que han realizado trabajos con polvos inertes, semejantes al de nuestra investigación, sobre otras plagas de almacenes.

Para estimar la toxicidad en cada uno de los tratamientos, se determinaron las siguientes variables:

Efecto anti-insecto: Para su evaluación, se adoptó la metodología propuesta por Lagunes \& Rodríguez (1989), la cual, se realizó mezclando polvo, a razón de $0,46 \mathrm{~g} ; 0,81 \mathrm{~g}$; $1,74 \mathrm{~g}$ con $35 \mathrm{~g}$ de semillas de café, debido al pequeño tamaño del insecto, en frascos plásticos estériles de $285 \mathrm{~g}$. Después de aplicada la marmolina a la muestra, se agitó bruscamente, para lograr una adhesión homogénea del polvo a las mismas. Luego, se procedió a su infestación, con 54 insectos adultos de $H$. hampei; esta variable, se determinó, para conocer el periodo de tiempo en que el polvo era capaz de matar los insectos y los efectos que causaba sobre los mismos y, para ello, se realizaron evaluaciones diarias.

La mortalidad, se evaluó a diario, después de realizada la infestación y se cuantificaron los insectos vivos y muertos en cada tratamiento y sus repeticiones.

Porcentaje de mortalidad: Se calculó utilizando la fórmula de Abbott (1925):

$$
\% M=\frac{\left(m_{e}-m b\right)}{(n-m b)} \times 100
$$

Donde:

$M=$ Mortalidad

me $=$ mortalidad en el tratamiento

$\mathrm{mb}=$ mortalidad en el blanco

$m_{e}=\frac{r}{n} \quad m_{b}=\frac{r^{\prime}}{n}$

$r=$ Insectos muertos en el tratamiento

$r^{\prime}=$ Insectos muertos en el blanco

$\mathrm{n}=$ Número de individuos

Porcentaje de pérdida de peso de los granos (Pp): Fue cuantificado, a partir de los 7 días de la infestación, contabilizando el número de semillas sanas y dañadas. Para su estimación, se utilizó la fórmula propuesta por Adams \& Schulten (1976) y mencionada por Silva et al. (2004):

$\mathrm{Pp}=100(\mathrm{Ngd} / \mathrm{Ntg}) \times \mathrm{C}$

Donde:

Pp es la pérdida de peso (\%)

Ngd: número de granos dañados

Ntg: número total de granos

C es 0,125 , si la semilla es almacenada como grano suelto.
Índice de germinación de las semillas: Se realizó una prueba de germinación de las semillas tratadas, para verificar si la marmolina influía en el poder germinativo. Para ello, se escogieron al azar 10 semillas por tratamiento de $35 \mathrm{~g}$, en cada repetición del experimento. Luego, se colocaron en placas de Petri con papel de filtro humedecido; éstas, fueron sometidas a un proceso de pre germinación durante 24 horas y se realizaron dos repeticiones por tratamiento en el tiempo.

Análisis estadístico: Todos los datos obtenidos fueron sometidos a análisis de varianza en el paquete estadístico Statgraphics para Windows versión 5.0. Para la mortalidad, todos los datos obtenidos fueron transformados en arc sen $(\mathrm{x} / 100)^{0,5}$. Los datos obtenidos en el porcentaje de germinación fueron sometidos a análisis de varianza.

\section{RESULTADOS Y DISCUSIÓN}

Durante la evaluación del efecto anti-insecto de la marmolina (Figura 1), se pudo comprobar que, a parir de las 12 horas de aplicado el polvo, causó efecto mortal sobre los insectos, alcanzando los mejores resultados a los tres días, con una dosis de $1,74 \mathrm{~g}$ y $0,81 \mathrm{~g}$, donde, a partir de este período de tiempo, se registraron los mayores niveles de muertes. Lo que difiere del control donde se registraron niveles de muertes muy inferiores a los encontrados en los tratamientos.

Estos resultados son superiores a los reportados por González Ferreira \& Gómez López (2006), quienes al utilizar tierra diatomeas en tres concentraciones $(0,1 \% ; 0,5 \% ; 1 \%)$, obtuvieron $1,67 \%$; $5,0 \%$ y $26,0 \%$ de insectos muertos, a los 10 días. Los resultados obtenidos en el presente trabajo difieren con el de los autores mencionados, debido a las concentraciones utilizadas por ellos, que en este estudio fueron superiores y difieren, también, en cuanto a la composición química de los compuestos utilizados, lo que pudo haber influido, directamente, sobre las muertes de los insectos.

La marmolina es un compuesto que se obtiene tras el corte y el procesamiento del bloque de mármol; es un polvo mineral, que posee químicamente $\mathrm{CaO}(55 \%), \mathrm{Ca} \mathrm{CO}_{3}(98,1 \%)$ y $\mathrm{MgO}$ (95\%), los cuales, tienen un efecto desecante abrasivo y actúan sobre el insecto, mediante un proceso físico de desecación abrasión, deshidratándolo y absorbiendo el agua de la cutícula cerosa externa, que protege al insecto de la desecación, al penetrar en las membranas inter-segmentales, después de entrar en contacto con los mismos. La alúmina y el sílice, nanoestructurada con composición química $\mathrm{AL}_{2} \mathrm{O}_{3}-$ $14 \%, \mathrm{SiO}_{2}-10 \%$, presente en el polvo, poseen características insecticidas; además, se caracterizan por partículas de 40$60 \eta \mathrm{m}$ y una superficie específica de $14 \mathrm{~m}^{2} \cdot \mathrm{g}^{-1}$, actuando sobre el insecto, en principio, sobre la base de carga electroestática 


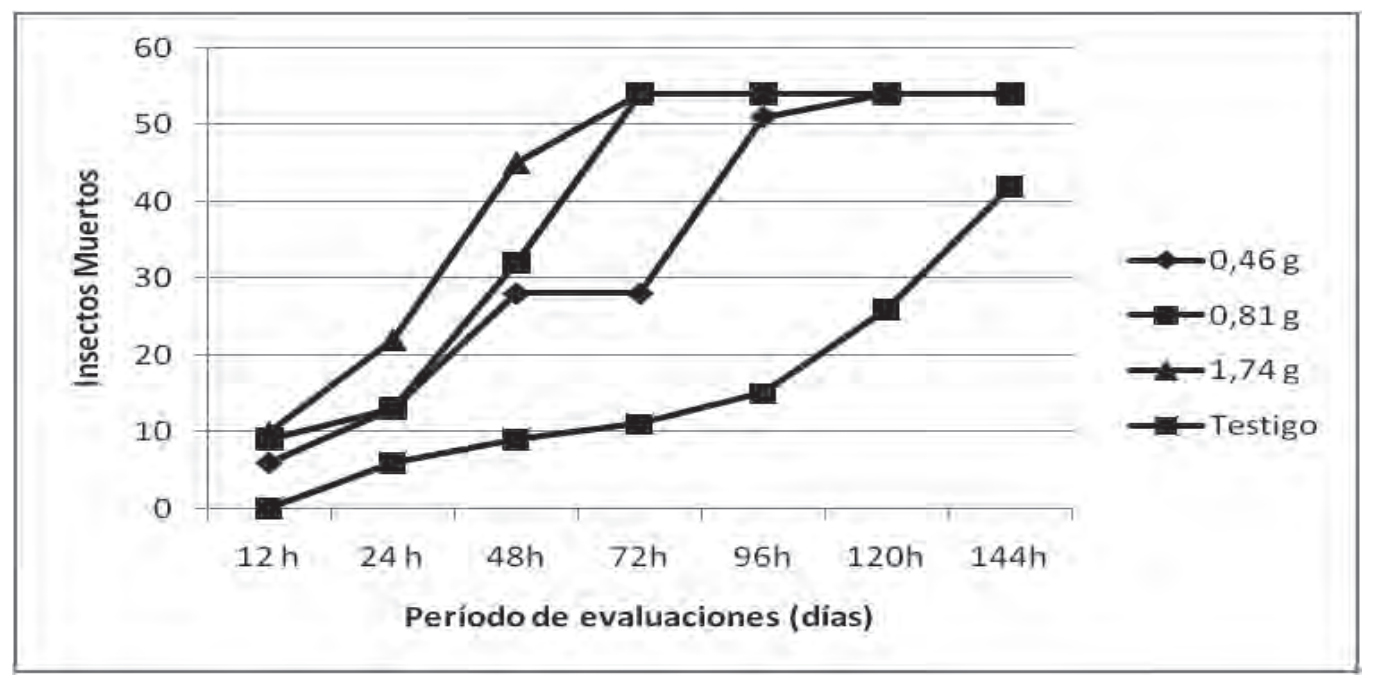

Figura 1. Efecto anti-insecto de la marmolina, a través del tiempo.

de las partículas y fenómenos de triboelectrificación (Stadler et al. 2010). Esto también coincide con Cook et al. (2008), quienes plantearon que las pequeñas partículas de polvo actúan a través de la absorción de las ceras cuticulares de los insectos.

En este trabajo, se obtuvo $100 \%$ de mortalidad, a partir de los 5 días de evaluación, con las tres dosis en estudio (Figura 2), lo que difiere, significativamente, con respecto al control, donde se registró un $37,7 \%$ de mortalidad, al término de los 15 días de evaluación.
Este resultado supera el obtenido por Silva et al. (2004), quienes utilizando una mezcla de Peumus boldus Molina:cal (50\%-50\%) al $1 \%$ de concentración sobre Sitophilus zeamais, obtuvieron un $97,7 \%$ de mortalidad de insectos y coincide con Pérez et al. (2012), quienes al comparar los efectos de PAO-2 ( $2 \mathrm{~g})$ y zeolita $(2 \mathrm{~g})$ sobre insectos en almacenes, obtuvieron $100 \%$ de mortalidad, a los 15 días, debido a que la zeolita y la tierra diatomeas, en principio, poseen compuestos similares al de esta investigación y son materiales minerales inertes; sin embargo, los resultados de

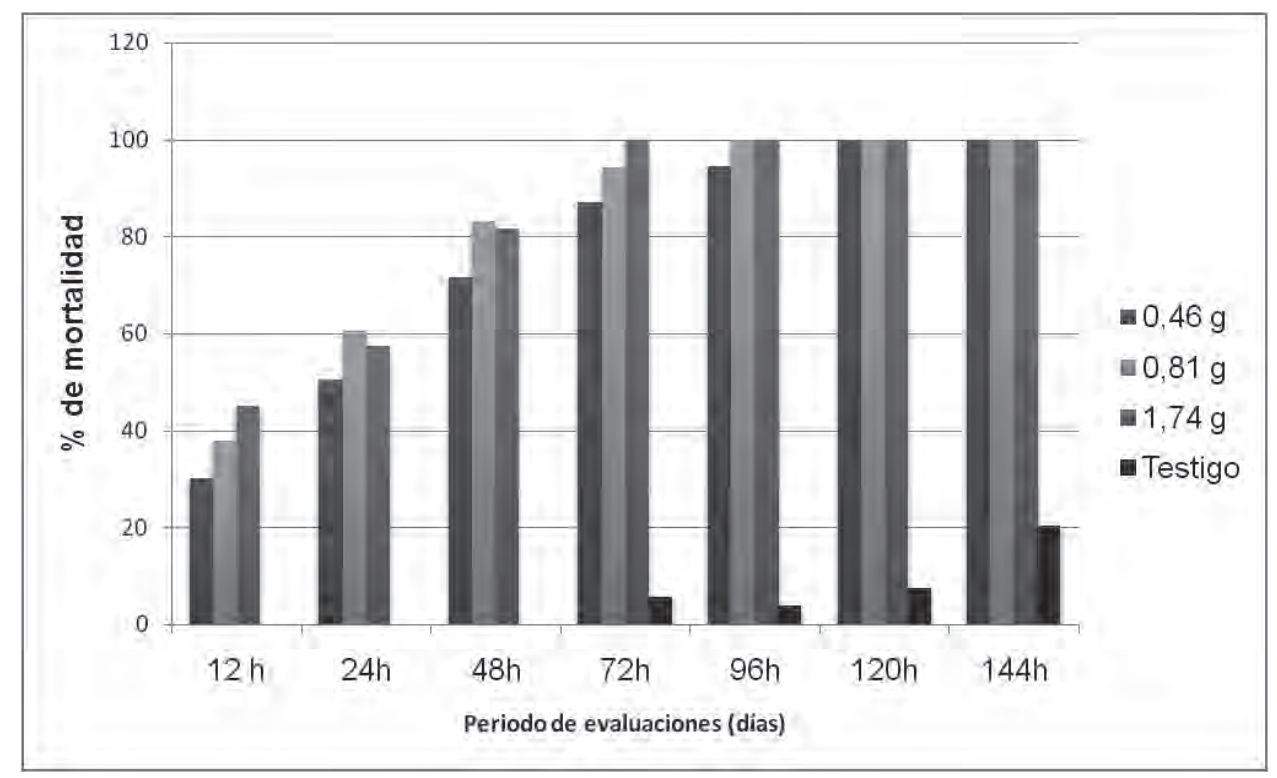

Figura 2. Mortalidad de los insectos, durante distintas etapas de evaluación. 
este estudio difieren con el autor mencionado anteriormente, en cuanto a la dosis utilizada, siendo la de esta investigación, de mejores resultados. Debemos destacar que Pérez et al. (2012) utilizaron una dosis de polvo zeolita más alta, que la utilizada en este estudio.

Supera los resultados obtenidos por Silva et al. (2004), quienes obtuvieron, con tierra de diatomeas, en sus tres concentraciones $(0,1 \% \mathrm{p} / \mathrm{p}, 1 \% \mathrm{p} / \mathrm{p}, 2 \% \mathrm{p} / \mathrm{p}), 76,9 \%, 92,6 \%$ y $98,8 \%$ de mortalidad, respectivamente, entre 21 y 28 días. Debido a la baja efectividad de la tierra diatomeas, por poseer menor número de compuestos químicos con respecto al polvo utilizado en esta investigación, lo que trae como consecuencia que, para lograr niveles de mortalidad aceptables en menor tiempo, se utilicen dosis más altas de tierra diatomeas, aspecto que no coincide con los de nuestro estudio, donde con dosis bajas, se obtienen resultados satisfactorios, en menor número de días.

También supera los resultados de este último autor, con diferentes concentraciones de carbonato de calcio $(1 \%$ y $2 \% \mathrm{p} / \mathrm{p}$ ), los cuales, causaron 70,2 y $84,2 \%$ de mortalidad: $31,8 \%$, con $\mathrm{CaO}$, a $0,1 \%$ p/p de concentración y $19,7 \%$ de mortalidad, con talco, al 1,0\% p/p de concentración, sobre insectos en almacenes. Los resultados obtenidos en nuestro trabajo difieren con el del autor, debido a las concentraciones utilizadas que, en este estudio, fueron superiores y difieren, también, en cuanto a la composición química de los compuestos. Debemos señalar que el autor mencionado anteriormente utilizó una dosis de un solo compuesto químico sobre el insecto y, en el caso de este trabajo, se aplicó una dosis compuesta con varios elementos químicos sobre el insecto, lo cual, sin dudas, influyó sobre la diferencia de resultados.

Durante el estudio, se observó que a las 4 horas después de estar en contacto $H$. hampei con la marmolina, comenzaron los efectos en el insecto, manifestando estos síntomas de pérdida de su capacidad de movimiento. También, se comprobó, tanto en los experimentos como en sus réplicas, que los insectos al morir manifestaban alas extendidas. Este hecho post mortem es causado por la afectación desecante de la marmolina a los músculos, específicamente, los de la locomoción, que salen del tórax, se disponen hacia la base de las alas y provocan la expansión y la retracción de las mismas.

Como lo planteado por Lagunes \& Vázquez (1994), un tratamiento prometedor debe ocasionar el $50 \%$ de mortalidad; según el criterio de este autor, se considera efectiva la marmolina en sus tres dosis $(0,46 \mathrm{~g} ; 0,81 \mathrm{~g}$; $1,74 \mathrm{~g}$ ), en el control de $H$. hampei, durante el periodo de conservación de semillas.
Pérdida de peso de las semillas (Pp): El tratamiento que menos pérdida de peso arrojó, se registró con una dosis de $1,74 \mathrm{~g}$, donde se determinó un valor $0,0 \% \mathrm{Pp}$, debido a que no se encontraron semillas afectadas, lo que significativamente difiere con respecto al testigo, donde se obtuvo un valor de 6,5\% Pp, al encontrarse 19 semillas afectadas del total utilizado.

Este resultado es superior al publicado por Pérez et al. (2012), quienes obtuvieron $2,34 \%$ de valor Pp, a una concentración de $1 \%(\mathrm{p} / \mathrm{p})$. Los resultados difieren, por la diferencia de concentraciones utilizadas por los autores, siendo más efectiva la marmolina, por el número de abrasivos que posee. Además, desde el punto de vista granulométrico, la marmolina es de menor tamaño de partícula, lo que posibilita que se adhiera mucho más a la superficie externa del insecto y también a la semilla de café, a la cual, el insecto afecta lo que influye en su protección.

No siendo así para las demás dosis utilizadas, donde los valores encontrados fueron de $1,8 \% \mathrm{Pp}$, con $0,46 \mathrm{~g}$ y $0,4 \%$ $\mathrm{Pp}$, con $0,81 \mathrm{~g}$, lo que arroja como resultado que entre mayor es la dosis de marmolina utilizada el valor del nivel de afectación a la semilla es más cercano a cero.

El análisis estadístico demostró una diferencia significativa entre las medias, con un nivel de significación de 5\% $(0,05 \%)$. Estadísticamente, los resultados arrojan que entre mayor número de insectos muertos menos será el número de semillas afectadas.

Germinación: La prueba de germinación, se realizó a los 10 meses de efectuados los tratamientos. El análisis de varianza ejecutado no mostró diferencia significativa (P-1.36364), debido a que el P-value no es inferior a 0,05; ninguno de los factores tiene efecto estadísticamente significativo en los tratamientos, para un nivel de confianza del 95,0\%, lo que significa que ninguna de las dosis utilizadas en el estudio afecta la germinación. Según se puede observar en la figura 3, los valores determinados en el control no difieren grandemente a los obtenidos en los tratamientos. Además, los valores obtenidos para todos los tratamientos están cercanos a $90 \%$ de germinación, que es un nivel muy próximo, al exigido para la exportación de semillas.

Resultados similares fueron obtenidos por Silva et al. (2004), quienes evaluaron cal, caolín, talco, tiza, carbonato de calcio, tierra de diatomeas y ceniza de carbón de espino, en la germinación de semillas de maíz. Los valores de germinación también estuvieron cercanos a los reportados por Pérez et al. (2012), quienes evaluaron la zeolita, como polvo inerte en la germinación de semillas de garbanzo. 


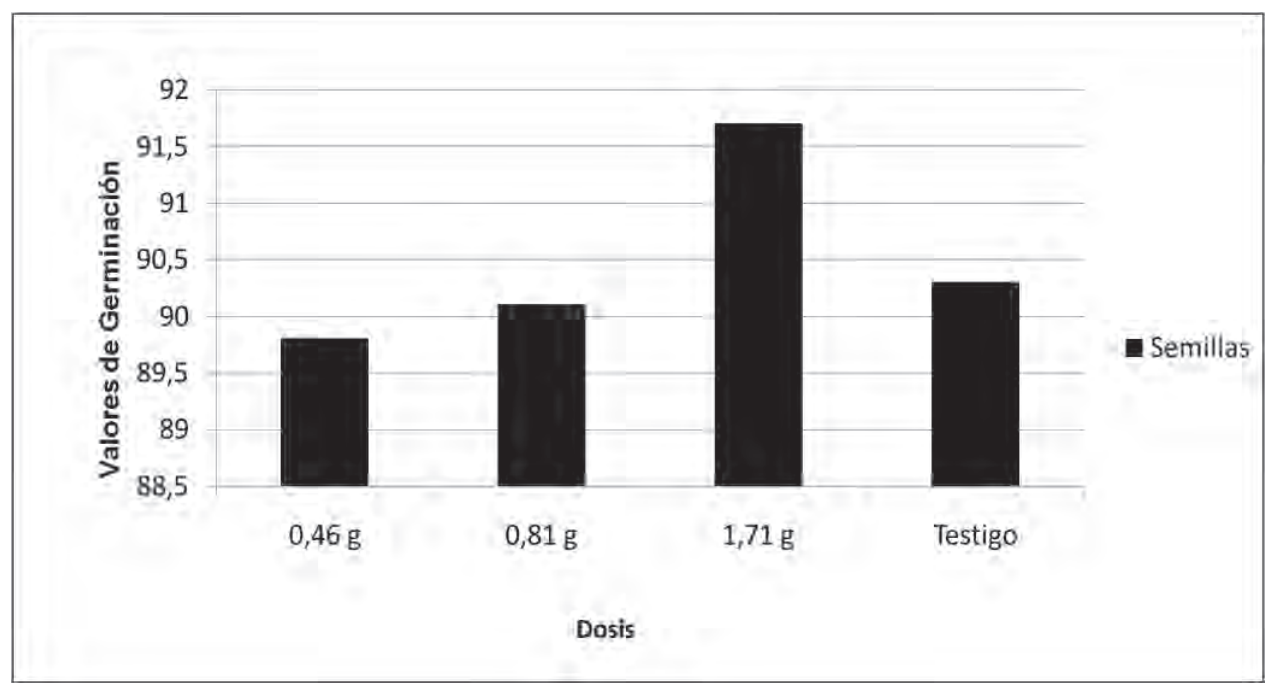

Figura 3. Porcentaje de germinación de semillas de café tratadas con marmolina, como polvo inerte.

La marmolina demuestra el poder anti-insecto sobre insectos coleópteros: Curculionidae, como material inerte, al disminuir la población de $H$. hampei, en semillas almacenadas.

Se evidencian las posibilidades de uso de este producto en el manejo de plagas en almacenes, provenientes de un material, abundantes en Cuba. El avance en esta investigación contribuye a reducir el empleo de insecticidas químicos, lo que es de especial importancia, en el mercado de productos orgánicos.

Es una alternativa, además, promisoria para el control de plagas de insectos, por tratarse de un producto natural; en cuanto a su composición química, eficaz, por la baja dosificación; seguro para el hombre y el ambiente, desde el punto de vista de su escasa reactividad química; con reducidas probabilidades de provocar resistencia a corto o mediano plazo, debido a su mecanismo de acción, basado en fenómenos físicos y no bioquímicos.

En la literatura consultada, no se encontraron referencias acerca de investigaciones en semillas de café ( $C$. arabica) con productos naturales, durante su conservación, ni tampoco del efecto de la marmolina sobre la broca del café (H. hampei); sin embargo, esta plaga fue susceptible a los efectos de este polvo. Elemento importante, debido a que es el primer estudio que se realiza (Cuba) para el control de la broca en semillas de café, en condiciones de almacenamiento.

Se puede agregar, además, que se consideró efectiva la marmolina en sus tres dosis $(0,46 \mathrm{~g} ; 0,81 \mathrm{~g} ; 1,74 \mathrm{~g})$, en el control de $H$. hampei, durante el período de conservación de café. Entre mayor la dosis de marmolina utilizada en las semillas menor fue el ataque del insecto a las mismas $y$, entonces, menor el porcentaje de pérdida de peso de la muestra.

Este polvo mineral aplicado a las semillas para el control de la broca del café no afectó la germinación de las semillas almacenadas, por un periodo de 10 meses.

Conflictos de intereses: Este trabajo fue elaborado y revisado con la participación de todos los autores, como equipo integrado de investigadores, quienes declaramos que no existe ningún conflicto de intereses que ponga en riesgo la validez de los resultados presentados. Financiación: Este estudio resultó del diagnóstico realizado durante la aplicación del Proyecto Agrocadena Alimentaria para la producción de granos en el Municipio Fomento, el cual, cuenta con financiamiento de la Agencia Suiza para el Desarrollo y la Cooperación (COSUDE).

\section{BIBLIOGRAFÍA}

1. ABBOTT, W.S. 1925. A method for computing the effectiveness of an insecticide. J. Econ. Entom. $18: 265-267$.

2. ADAMS, J.M.; SCHULTEN, G.G.M. 1976. Losses caused by insects, mites and microorganisms. In: Harris, K.L.; Lindblad, C.J. (Compiladores). Postharvest grain loss assessment methods. AACC. p.83-93. 
3. ARIZALETA, M.; PIRE, R.; PARÉS, J. 2002. Efecto de la fertilización con N-P-K sobre el contenido foliar y el crecimiento del cafeto (Coffea arabica L.) en etapa de vivero, en la población de Villanueva, Estado Lara, Venezuela. Rev. Café y Cacao. 3(2):57-61.

4. ARIZALETA, M.; MONTILLA, J.; PARES, J. 2005. Efecto del almacenamiento de las semillas de cafeto (Coffea arabica L. var. Catuai amarrillo) sobre la emergencia. Rev. Fac. Agron. 22(3). Disponible desde Internet en: http://www.scielo.org.ve/scielo.php?pid=S0378$78182005000300001 \&$ script $=$ sci_arttext $\quad$ (con acceso 21/01/2015).

5. ARRUBLA, P.F.; CÁRDENAS, M.; POSADA, F.J. 2009. Adherencia de las esporas de Beauveria bassiana formuladas en polvo y líquido sobre la broca del café. Rev. U.D.C.A Act. \& Div. Cient. 11(1):123-133.

6. BARBOZA, R.; HERRERA, J. 1990. El vigor en la semilla de café y su relación con la temperatura de secado, el contenido de humedad y las condiciones de almacenamiento. Agr. Costarricense. 14(1):1-8.

7. BUSTILLO-PARDEY, A.E. 2006. Una revisión sobre la broca del café, Hypothenemus hampei, en Colombia. Rev. Col. Ent. 32(2):101-116.

8. BUSTILLO, A.E. 2005. El papel del control biológico en el manejo integrado de la broca del café Hypothenemus hampei (Ferrari) (Coleoptera: Scolytidae). Rev. Acad. Col. Cienc. Exac. Fís. Nat. 29(110):55-69.

9. BUSTILLO, A.E.; BENAVIDES, M.P.; OROZCO, H.J.; POSADA, F.J. 2008. Manejo Integrado de la broca del Café Hipothenemus hampei (Ferrari) en Colombia. Caldas, Chinchiná, Cenicafé, 134p.

10. CINTRÓN-VALDÉS, B.; GRILLO-RAVELO, H. 2009. Dinámica poblacional de Hypothenemus hampei (Ferrari) (Coleoptera: Curculionidae) durante la postcosecha del café en Topes de Collantes, y Jibacoa. Centro Agrícola. 36(2):71-76.

11. COOK, D.A.; WAKEFIELD, M.E.; BRYNING, G.P. 2008. The physical action of three diatomaceous earths against the cuticle of the flour mite Acarus siro L. (Acari: Acaridae). Pest Manag. Sci. 64:141-146.

12. DÍAS, M.; BARRIOS, A. 1993. Conservaçaô de sementes de café (Coffea arabica L.) en diferentes embalajes. Bragantia. 15(2):197-202.
13. DÍAZ-VICENTE, J.M.; PÉREZ-QUINTANILLA, J.N.; MAGALLANES, R.; CEDEÑO, R.; PINSONRINCÓN, E.P.; DE COSS-FLORES, M.E.; CABRERAALVARADO, M.E. 2014. Control biológico de la broca del café Hypothenemus hampei Ferrari (Coleoptera: Curculionidae) con diferentes dosis del hongo Beauveria bassiana (Bals.) Vuill. (Moniliales: Moniliaceae) en Unión Juárez, Chiapas, México. Vedalia. 15:15-21.

14. STADLER, T.; BUTELER, M.; WEAVER, D.K. 2010. Nanoinsecticidas: Nuevas perspectivas para el control de plagas. Rev. Soc. Entomol. Argent. 69(34):149-156.

15. GONZÁLEZ FERREIRA, M.L.; GÓMEZ LÓPEZ, V. 2006. Evaluación de la mortalidad de adultos de Sitophilus zeamays (Coleoptera: Curculionidae) ocasionada por diferentes concentraciones de Tierra de Diatomeas y pimienta negra (Piper nigrum) en maíz almacenado. Invest. Agr. (Paraguay). 8(2):45-49.

16. LAGUNES, T.A.; RODRÍGUEZ, J.C. 1989. Grupos toxicológicos de insecticidas y acaricidas. In: Temas selectos de manejo de insecticidas agrícolas. (Tomo 1), CONACYT/Montecillo, Edo. México. p.24-106.

17. LAGUNES, T.A.; VÁZQUEZ, N.M. 1994. El Bioensayo en el Manejo de Insecticidas y Acaricidas. CONACYT / Montecillo, Edo. de México. 159p.

18. LEVY, F.; FASUOLI, I.; GALLO, P. 1989. Estudios de germinacao de sementes de Coffea canephora armazenadas en diferentes condices. Instituto Brasileiro do Café. Directora de Producto. p.159160.

19. ORTUÑO, F.; ECHANDI, Z. 1980. Efecto de condiciones de almacenamiento sobre la viabilidad y vigor de la semilla de café (Coffea arabica L.). Agron. Costarricense. 4(2):149-159.

20. PÉREZ, J.C.; ORIELA, P.; RAMÍREZ, S.; SURIS, M. 2012. Evaluación de productos naturales para el control de Lasioderma serricorne (F.) (Coleoptera: Anobiidae) sobre garbanzo (Cicer arietinum L.) en condiciones de laboratorio. Rev. Protección Veg. 27(1). Disponible desde Internet en: http:// scielo.sld.cu/scielo.php?script $=$ sci_arttext\&pi $\mathrm{d}=\mathrm{S} 1010-27522012000100005 \quad$ (con acceso 14/01/2015).

21. PÉREZ, N.; CASTELLANOS, L.; JIMÉNEZ, R. 2013. Impacto de la entrada de la broca del café 
(Hypothenemus hampei Ferrari) en la Empresa Municipal agropecuaria de Fomento. Rev. Centro Agrícola. 40(1):5-9.

22. QUISPE-CONDORI, R.; LOZA-MURGUIA, M.; MARZAMAMANI, F.; GUTIÉRREZ, R.; RIQUELME, C.; ALIAGA, F.; FERNÁNDEZ, C. 2014. Trampas artesanales con atrayentes alcohólicos en el control de la broca del café, Hypothenemus hampei (Ferrari 1867) en la Colonia Bolinda, Caranavi. J. Selva Andina Biosphere. 3(1):2-14.

23. SILVA, G.; GONZÁLEZ, P.; HEPP, R.; CASALS, P.; BUSTOS, T. 2004. Control de Sitophilus zeamais Motschulsky con polvos inertes. Agrociencia. 38(5):529-536. Disponible desde Internet en: www.redalyc.org/ revista.oa?id=302 (con acceso 28/01/2015).
24. URRIBARRÍ, A.; ZABALA, A.; SÁNCHEZ, J.; ARENAS, E.; CHANDLER, C.; RINCÓN, M.; GONZÁLEZ, E.; AIELLO, C.M. 2014. Evaluación del potencial de la borra de café como materia prima para la producción de biodiesel. Rev: Multiciencias. 14(2):129.139. Disponible desde Internet en: http://www.redalyc.org/ pdf/904/90432601006.pdf (con acceso 02/06/2015).

25. VÁZQUEZ, L.L.; GARCÍA, R.; PEÑA, E. 2005. Observaciones sobre la presencia de brocas del café (Hypothenemus hampei) en los frutos que caen al suelo. Rev. Fitosanidad. (Cuba). 9(2):47-48.

Recibido: Febrero 2 de 2015

Aceptado: Septiembre 7 de 2015

\section{Cómo citar:}

Rodríguez Ledesma, Y.; Suárez Pérez, C.; Rojas Rojas, J.A.; Mirabal García, R.; López López, Y.; Molina Moreno, E. 2015. Efectividad de la marmolina como mineral inerte en el control de Hypothenemus hampei (Ferrari) (Coleoptera: Curculionidae) durante la conservación de semillas. Rev. U.D.C.A Act. \& Div. Cient.18(12): 351-358. 